\title{
Analytical Solution of Non-Newtonian Nanofluid Flows Within Circular Duct under Convective Boundary Condition
}

\author{
Antar Tahiri ${ }^{1,2 *}$, Kacem Mansouri ${ }^{2}$, Kouider Rahmani $^{3}$, Amar Kouadri $^{4}$, Embarek Douroum $^{5}$ \\ ${ }^{1}$ Laboratory of Development in Mechanics and Materials, LDMM, University of Djelfa, Djelfa 17000, Algeria \\ ${ }^{2}$ Energy and Mechanical Engineering Laboratory, University M. Bougara of Boumerdes, Boumerdes 35000, Algeria \\ ${ }^{3}$ Modeling Simulation and Optimization of Real Complex Systems Laboratory, University of Djelfa, Djelfa 17000, Algeria \\ ${ }^{4}$ Gas Turbine Joint Research Team, University of Djelfa, Djelfa 17000, Algeria \\ ${ }^{5}$ Laboratory of materials and reactive systems, LMSR, Djillali Liabes University, Sidi Bel Abbes 22000, Algeria
}

Corresponding Author Email: antartahiri@yahoo.fr

https://doi.org/10.18280/mmep.080504

Received: 23 August 2020

Accepted: 14 September 2021

\section{Keywords:}

variational method, convective boundary conditions, non-Newtonian nanofluids, viscous dissipation, circular duct

\begin{abstract}
At the outset, this work aims to carried out an analytical investigation of forced convection by establishing a laminar flow into circular duct under convective boundary conditions of the third type for non-Newtonian nanofluid, the fluid containing $\mathrm{TiO} 2$ uniformly dispersed in aqueous solution with $0.5 \mathrm{wt} \%$ of $\mathrm{CMC}$ solutions (Carboxymethyl Cellulose) is used as working fluid. The viscous dissipation effects are taken into account, the employed methodology is based on a combination of the Ritz variational approach with the Laplace transformation technique, so the power-law fluids flow model is used to describe the non-Newtonian fluid behavior. The effect of dimensionless parameters such as Biot $(B i)$, Brinkman $(B r)$, Peclet $(P e)$ numbers, power-law index $(n)$, and nanoparticles concentration $(\varphi)$ on the temperature distribution contours and on the examined local Nusselt number. Our results have been compared with those found in the literature in particular the cases of base fluids $(\varphi=0)$ with and without viscous dissipation effects.
\end{abstract}

\section{INTRODUCTION}

The importance of convective heat transfer in conduits and their industrial uses, including cooling or heating of various technical equipment used in industry, for example electronic devices, renewable heat recovery energies, heat exchangers used in different systems. Many of analytical, numerical and empirical studies have been conducted.

Today, the potential for energy demand is increasing and to improve the performance and the efficiency of thermal devices, several solutions have been considered. Among the solutions proposed to improve the heat transfer rate, either by increasing the heat exchange surface by adding internal or external fins $[1,2]$, or the use of corrugated tubes [3] and elliptical tubes [4]. However, the fluids used in the heat transfer fields such as water, ethylene glycol, and motor oil have weak thermal conductivity compared to solid materials. The addition of solid nanoparticles $\left(\mathrm{Al}_{2} \mathrm{O}_{3}, \mathrm{CuO}, \mathrm{TiO}_{2}, \mathrm{Cu}\right.$, etc. $)$ to a base fluid improves considerably the thermal conductivity of the resulting fluid called the nanofluid [5]. During the last decade, many experimental and numerical studies have been carried out.

Numerous studies have shown a significant improvement in the thermal efficiency of the mixing [6-11]. An experimental study has been performed by Lee et al. [12], they showed that nanofluids with small quantity of nanoparticles have thermal conductivity higher than the same fluid without nanoparticles. They found that an improvement of $20 \%$ in the thermal conductivity of the copper oxide/ethylene glycol nanofluid is observed for the case of the volume fraction $(0.04 \%)$. Heris et al. [13] have carried out an experimental investigation to study the influence of the addition of copper oxide and alumina nanofluids on the laminar heat flow in circular duct under constant wall temperature, with water considered as a base fluid. Heris et al. [14] concluded that the increase in thermal conductivity is not the only factor which improve the nanofluids heat transfer, moreover other parameters such as the nanoparticles dispersion and the Brownian motion etc..., can affect the heat transfer improvement.

In several works, nanoparticles are added to a Newtonian base fluid, and the resulting fluid behaves as Newtonian fluid. In other works, base fluids with Newtonian and nonNewtonian behaviors have been used [15-18]. Santra et al. [15], in their numerical study, they have used a nanofluid composed of copper nanoparticles dispersed in the water for a large range of Reynolds number (5-1500) with different values of volume fraction $(0.00<\varphi<0.050)$. It has been shown that for the case of non-Newtonian nanofluids, the wall shear stress increases clearly with the increase of the volume fraction for low Reynolds numbers.

Hojjat et al. $[16,17]$ have studied experimentally the forced convection heat transfer of non-Newtonian nanofluid composed of an aqueous solution of $0.5 \mathrm{wt} \%$ of CMC solutions (Carboxymethyl Cellulose) and nanoparticles of $\gamma-\mathrm{Al}_{2} \mathrm{O}_{3}$, $\mathrm{CuO}$ and $\mathrm{TiO}_{2}$. Their obtained results showed that the average and local heat transfer coefficients of nanofluids are higher than those of the base fluids. In addition, the length of the thermal establishment flow of nanofluids is greater than that of the base fluids, where it increases with the increase of the nanoparticles concentration. An experimental study shows 
that the rheological behavior of the nanofluids described above at different volume concentrations of nanoparticles exhibits pseudo plastic behavior (thinning by shearing) [18]. Yang et al. [19] have compared experimentally the thermal properties such as conductivity and viscosity of nanofluid composed of distilled water and copper nanoparticles, with another nanofluid composed of aqueous solution of cetyl trimethyl ammonium chloride/sodium-salicylate (viscoelastic fluid) under the same operating conditions. Their results showed that the nanofluid has a higher thermal conductivity than that composed of water and copper nanoparticles, and that its thermal conductivity increases with the increase of the volume fraction of the nanoparticles. Bahiraei et al. [20] have studied numerically the flow and heat transfer of non-Newtonian nanofluids composed of $\mathrm{CuO}$ nanoparticles dispersed in the $0.5 \mathrm{wt} \%$ aqueous solution of CMC (Carboxy Methyl Cellulose) with volume fraction of $0<\varphi<0,04 \%$. The nanofluid flows between two parallel plates constituting a microchannel with a constant temperature imposed on the wall. The results indicated that with the baffles, the increase of Reynolds number and of volume fraction of nanoparticles enhance the thermal behavior of the non-Newtonian nanofluid. However, in some cases, the shear thickening behavior is observed when the nanoparticles increase. Tahiri and Mansouri [21, 22] have studied analytically the convective heat transfer using nonNewtonian nanofluid within circular duct, taking into consideration the effect of viscous dissipation. It's demonstrated that the non-Newtonian nanofluids show a better efficiency in term of heat transfer compared to the Newtonian nanofluids. Recently, Ragueb and Mansouri [23] have investigated numerically the laminar non-Newtonian nanofluid flows within elliptical ducts under convective boundary conditions at the wall (third kind), without effect of viscous dissipation. The obtained results showed an improvement in the heat transfer coefficient when rising the volume fractions and decreasing the form factor.

In the most studies mentioned above, heat transfer problems of the nanofluids are solved numerically or investigated experimentally. In this work, the analytical solution for nonNewtonian nanofluid composed of $\mathrm{TiO}_{2}$ nanoparticles dispersed in $0.5 \mathrm{wt} \% \mathrm{CMC}$ solutions, flowing within circular duct and under third kind boundary conditions is investigated analytically by considering the effect of viscous dissipation. The asymptotic values of Nusselt number for the first and second kind boundary conditions are estimated in particular cases when Biot number tends to infinity and zero respectively.

\section{MATHEMATICAL MODEL}

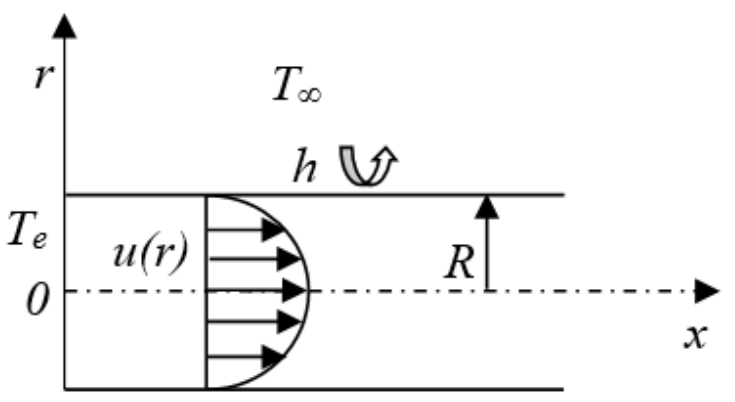

Figure 1. Description of the physical domain

Laminar flow of non-Newtonian nanofluid $\left(\mathrm{TiO}_{2} / 0.5 \mathrm{wt} \%\right.$
CMC) within circular duct is investigated analytically, assuming that it's dynamically steady, fully developed and thermally evolving (Figure (1)). The axial thermal conduction is simply assumed to be negligible. The rheological behavior of the current non-Newtonian fluids can be defined by the power-law model.

\subsection{Governing equations}

The energy equation in cylindrical coordinates is written by:

$$
\left(\rho c_{p}\right)_{n f} u(r) \frac{\partial T}{\partial x}=\frac{1}{r} \frac{\partial}{\partial r}\left(k_{n f} r \frac{\partial T}{\partial r}\right)+\tau_{r x} \frac{\partial u}{\partial r}
$$

The boundary conditions can be expressed as:

$$
\begin{gathered}
T(x=0, r)=T_{e} \\
\frac{\partial T(x, r=0)}{\partial r}=0 \\
k_{n f} \frac{\partial T(x, r)}{\partial r}+h_{e}\left(T(x, r)-T_{\infty}\right)=0, r=R, x>0
\end{gathered}
$$

Cotta and Özişik [24] give the velocity profile $u(r)$ and stress tensor $\tau_{r x}$ for a fully developed laminar flow in a circular duct as follows:

$$
\begin{gathered}
\tau_{r x}=m\left|\frac{\partial u}{\partial r}\right|^{n-1} \cdot \frac{\partial u}{\partial r} \\
u(r)=\frac{3 n+1}{n+1} u_{a v}\left[1-\left(\frac{r}{R}\right)^{\frac{n+1}{n}}\right]
\end{gathered}
$$

where, the average velocity of flow, the power-law index and the consistency factor are $u_{a v}, n$ and $m$, respectively. Remember that for Newtonian fluid $n=1$, when $n$ is in the range $0<n<1$, the fluid is said to be pseudo-plastic (or shear thinning) and when $n>1$, the fluid is said to be dilatant (or shear thickening). By substituting Eqns. (5) and (6) into Eq. (1), we obtain:

$$
\begin{aligned}
& \left(\rho c_{p}\right)_{n f} u(r) \frac{\partial T(x, r)}{\partial x} \\
& =k_{n f} \frac{1}{r} \frac{\partial}{\partial r}\left[r \frac{\partial T(x, r)}{\partial r}\right]+m\left(\frac{3 n+1}{n}\right)^{n+1}\left(\frac{u_{a v}}{R}\right)^{n+1}\left(\frac{r}{R}\right)^{\frac{n+1}{n}}
\end{aligned}
$$

Let us define the dimensionless quantities in the form:

$$
\begin{aligned}
& x^{+}=\frac{x}{P e_{n f} R}, r^{+}=\frac{r}{R}, \theta=\frac{T-T_{\infty}}{T_{e}-T_{\infty}},\left(\Delta T=T_{e}-T_{\infty}\right) \\
& P e_{n f}=\frac{(3 n+1) u_{a v}^{(n+1)} R(\rho C p)_{n f}}{(n+1) K_{n f}}, B i=\frac{h_{e} D_{h}}{K_{n f}}, B r=\frac{m\left(u_{a v}\right)^{n+1}}{\Delta T k_{n f} D_{h}^{n-1}}
\end{aligned}
$$

The system Eq. (1) to Eq. (4) will be rewritten in the following dimensionless equations: 


$$
\begin{gathered}
{\left[1-\left(r^{+}\right)^{\frac{n+1}{n}}\right] \frac{\partial \theta}{\partial x^{+}}=\frac{1}{r^{+}} \frac{\partial}{\partial r^{+}}\left(r^{+} \frac{\partial \theta}{\partial r^{+}}\right)+} \\
2^{n-1} B r\left(\frac{3 n+1}{n}\right)^{n+1}\left(r^{+}\right)^{\frac{n+1}{n}} \\
\theta\left(x^{+}, r^{+}\right)=1, \quad x^{+}=0 \\
\frac{\partial \theta\left(x^{+}, r^{+}=0\right)}{\partial r^{+}}=0, \quad x^{+}>0 \\
\frac{\partial \theta\left(x^{+}, r^{+}\right)}{\partial r^{+}}+\frac{1}{2} B i . \theta=0, \quad r^{+}=1, \quad x^{+}>0
\end{gathered}
$$

\subsection{Method of solution}

The governing Eq. (8) and the condition (9) are nonhomogeneous. Hence, the resolution of the system (Eqns. (8) to (11)) can be formulated as follows by linear superposition:

$$
\theta\left(x^{+}, r^{+}\right)=\theta_{1}\left(x^{+}, r^{+}\right)+\theta_{\infty}\left(r^{+}\right)
$$

where, the function $\theta_{1}$ represents the temperature distribution within the thermally developing zone. This function can be expressed in the fully developed zone as following:

$$
\lim _{x^{+} \rightarrow \infty} \theta\left(x^{+}, r^{+}\right)=\theta_{\infty}\left(r^{+}\right)
$$

and

$$
\theta_{1}\left(x^{+}, r^{+}\right)=0
$$

Substituting Eq. (13) into Eq. (8) offers the ordinary differential equation of $\theta_{\infty}\left(r^{+}\right)$as:

$$
\begin{gathered}
\frac{1}{r^{+}} \frac{\partial}{\partial r^{+}}\left(r^{+} \frac{\partial \theta_{\infty}}{\partial r^{+}}\right)+2^{n-1} B r\left(\frac{3 n+1}{n}\right)^{n+1}\left(r^{+}\right)^{\frac{n+1}{n}}=0 \\
\frac{\partial \theta_{\infty}\left(r^{+}=0\right)}{\partial r^{+}}=0
\end{gathered}
$$$$
\frac{\partial \theta_{\infty}\left(r^{+}\right)}{\partial r^{+}}+\frac{1}{2} \text { Bi. } \theta_{\infty}\left(r^{+}\right)=0, \quad r^{+}=1
$$

with respect to $r^{+}$, this system may be integrated twice, and the solution obtained is:

$$
\theta_{\infty}\left(r^{+}\right)=2^{n-1} B r\left(\frac{3 n+1}{n}\right)^{n-1}\left(1-r^{+\frac{3 n+1}{n}}\right)+2^{n} \frac{B r}{B i}\left(\frac{3 n+1}{n}\right)^{n}
$$

The next step is the calculation of the temperature distribution $\theta_{1}\left(x^{+}, r^{+}\right)$in the entrance region:

$$
\left[1-\left(r^{+}\right)^{\frac{n+1}{n}}\right] \frac{\partial \theta_{1}}{\partial x^{+}}=\frac{1}{r^{+}} \frac{\partial}{\partial r^{+}}\left(r^{+} \frac{\partial \theta_{1}}{\partial r^{+}}\right)
$$

$$
\begin{gathered}
\theta_{1}\left(x^{+}=0, r^{+}\right)=1-\theta_{\infty} \\
\frac{\partial \theta_{1}\left(x^{+}, r^{+}=0\right)}{\partial r^{+}}=0, \quad x^{+}>0 \\
\frac{\partial \theta_{1}\left(x^{+}, r^{+}\right)}{\partial r^{+}}+\frac{1}{2} \text { Bi. } \theta_{1}=0, \quad r^{+}=1, \quad x^{+}>0
\end{gathered}
$$

By applying Laplace transformation of the dimensionless temperature, we obtain:

$$
\overline{\theta_{1}}\left(s, r^{+}\right)=\int_{0}^{\infty} \exp \left(-s x^{+}\right) \theta_{1}\left(x^{+}, r^{+}\right) d x^{+}
$$

The Laplace transforms properties for Eqns. (19) to (22) yield:

$$
\begin{gathered}
\frac{1}{r^{+}} \frac{\partial}{\partial r^{+}}\left(r^{+} \frac{\partial \bar{\theta}_{1}}{\partial r^{+}}\right)+u^{+}\left(r^{+}\right)\left[\left(1-\theta_{\infty}\right)-s \bar{\theta}_{1}\right]=0 \\
\frac{\partial \overline{\theta_{1}}\left(s, r^{+}=0\right)}{\partial r^{+}}=0 \\
\frac{\partial \bar{\theta}_{1}\left(s, r^{+}\right)}{\partial r^{+}}+\frac{1}{2} B i \cdot \overline{\theta_{1}}=0, r^{+}=1
\end{gathered}
$$

The previous problem can be readily solved by Ritz method, and the following expression gives the corresponding variational form:

$$
\begin{gathered}
I=\int_{0}^{1}\left[\left(\frac{\partial \overline{\theta_{1}}}{\partial r^{+}}\right)^{2}+s u^{+}\left(r^{+}\right) \bar{\theta}_{1}^{2}-2 u^{+}\left(r^{+}\right)\left(1-\theta_{\infty}\right) \overline{\theta_{1}}\right] r^{+} d r^{+} \\
+\left.\frac{1}{2} B i \cdot \bar{\theta}_{1}^{2}\right|_{r^{+}=1}
\end{gathered}
$$

The first step of this analytical approach is to choose a preliminary solution which must satisfy the boundary conditions. The solution is approximated by series of function $\psi_{i}$ :

$$
\overline{\theta_{1}}\left(s, r^{+}\right)=\sum_{i=0}^{N} c_{i}(s) \psi_{i}\left(\sigma_{i} r^{+}\right)
$$

where, the $i^{\text {th }}$ function $\psi_{i}\left(\sigma_{i} r^{+}\right)$is given by:

$$
\psi_{i}\left(\sigma_{i} r^{+}\right)=M\left(a, b, \sigma_{i} r^{+2}\right) \exp \left(-\sigma_{i} \frac{r^{+2}}{2}\right), \quad a=\frac{2-\sigma_{i}}{4}, b=1
$$

The confluent hypergeometric function $M\left(a, b, \sigma_{l} r^{+2}\right)$ is defined as:

$$
\begin{gathered}
M\left(a, b, \sigma_{i} r^{+2}\right)=1+\frac{a}{b}\left(\sigma_{i} r^{+2}\right)+\frac{a(a+1)}{b(b+1) 2 !}\left(\sigma_{i} r^{+2}\right)^{2} \\
+\frac{a(a+1)(a+2)}{b(b+1)(b+2) 3 !}\left(\sigma_{i} r^{+2}\right)^{3}+\ldots
\end{gathered}
$$


Using the iterative Newton's method, the eigenvalues $\left(\sigma_{i}\right)$ are the roots of the transcendental Eq. (30):

$$
\begin{gathered}
\left.\sigma_{i} \cdot \exp \left(-\frac{\sigma_{i}}{2}\right)\left[\frac{2 a}{b} M(a+1, b+1), \sigma_{i}\right)-M\left(a, b, \sigma_{i}\right)\right]+ \\
\frac{1}{2} \operatorname{Bi} \cdot M\left(a, b, \sigma_{i}\right) \exp \left(-\frac{\sigma_{i}}{2}\right)=0
\end{gathered}
$$

Including these trial solutions $\psi_{i}\left(\sigma_{i} r^{+}\right)$into the expression (27) and implies that:

$$
\frac{\partial I\left(C_{i}\right)}{\partial C_{i}}=0 ; \quad i=1,2, \ldots N
$$

The algebraic equations for the system can be expressed as:

$$
\left\{\begin{array}{l}
a_{11} C_{1}(s)+\ldots+a_{1 N} C_{N}(s)=b_{1} \\
\cdots \\
a_{N 1} C_{1}(s)+\ldots+a_{N N} C_{N}(s)=b_{N}
\end{array}\right.
$$

and in matrix form as:

$$
[A][C]=[B]
$$

where, the symmetric square matrix $[A]$ can be written as:

$$
[A]=\left[A^{*}\right]+s[D]
$$

The matrix elements $\left(a_{i j}^{*}\right)$ and $\left(d_{i j}\right)$ and are given, respectively:

$$
\begin{gathered}
a_{i j}^{*}=\int_{0}^{1} \frac{\partial \psi_{i}}{\partial r^{+}} \frac{\partial \psi_{j}}{\partial r^{+}} r^{+} d r^{+}+\frac{1}{2} B i \cdot \psi_{i}\left(r^{+}=1\right) \cdot \psi_{j}\left(r^{+}=1\right) \\
a_{i j}^{*}=\int_{0}^{1} \sigma_{i} \sigma_{j} r^{+2} \exp \left(-\sigma_{i} \frac{r^{+2}}{2}\right) \exp \left(-\sigma_{j} \frac{r^{+2}}{2}\right) \\
{\left[\frac{2 a \sigma_{i}}{b} M\left(a+1, b+1, \sigma_{i} r^{+2}\right)-M\left(a, b, \sigma_{i} r^{+2}\right)\right]} \\
\times\left[\frac{2 a \sigma_{j}}{b} M\left(a+1, b+1, \sigma_{j} r^{+2}\right)\right. \\
\left.\quad-M\left(a, b, \sigma_{j} r^{+2}\right)\right] r^{+} d r^{+} \\
\quad=1) ; \quad i, j=1,2, \ldots N
\end{gathered}
$$

To calculate the values of integrals in Eqns. (35), (36) and (37), we use the numerical Simpson's method.

The vector $[B]$ is given by:

$$
b_{i}=\int_{0}^{1}\left(1-\left(r^{+}\right)^{\frac{n+1}{n}}\right)\left(1-\theta_{\infty}\right) M\left(a, b, \sigma_{i} r^{+2}\right) \exp \left(-\sigma_{i} \frac{r^{+2}}{2}\right) r^{+} d r^{+}
$$

Introducing Eq. (34) into Eq. (33), we find the coefficients $C_{i}(\mathrm{~s})$ :

$$
C_{i}=\frac{\beta_{i}}{s+\lambda_{i}}
$$

where, $\beta_{i}$ and $\lambda_{i}$ are respectively the elements of the vector $[A][D]^{-1}$ and the eigenvalues of the matrix $\left[A^{*}\right][D]^{-1}$.

The dimensionless temperature can be written in Laplace domain as follows:

$$
\bar{\theta}_{1}\left(r^{+}, s\right)=\sum_{i=1}^{N} \frac{\beta_{i}}{s+\lambda_{i}} M\left(a, b, \sigma_{i} r^{+2}\right) \exp \left(-\sigma_{i} \frac{r^{+2}}{2}\right)
$$

the inverse transform of Eq. (40) can be written as:

$$
\theta_{1}\left(x^{+}, r^{+}\right)=\sum_{i=1}^{N} \beta_{i} M\left(a, b, \sigma_{i} r^{+2}\right) \exp \left(-\sigma_{i} \frac{r^{+2}}{2}\right) \exp \left(-\lambda_{i} x^{+}\right)
$$

Finally, the general solution of our problem can be expressed:

$$
\begin{aligned}
\theta\left(x^{+}, r^{+}\right)= & \sum_{i=1}^{N} \beta_{i} M\left(a, b, \sigma_{i} r^{+2}\right) \exp \left(-\sigma_{i} \frac{r^{+2}}{2}\right) \exp \left(-\lambda_{i} x^{+}\right)+ \\
& 2^{n-1} B r\left(\frac{3 n+1}{n}\right)^{n-1}\left(1-r^{+\frac{3 n+1}{n}}\right)+2^{n} \frac{B r}{B i}\left(\frac{3 n+1}{n}\right)^{n}
\end{aligned}
$$

The wall temperature is given by:

$$
\begin{gathered}
\theta_{w}=\theta\left(x^{+}, 1\right) \\
=\sum_{i=1}^{N} \beta_{i} M\left(a, b, \sigma_{i}\right) \exp \left(-\frac{\sigma_{i}}{2}\right) \exp \left(-\lambda_{i} x^{+}\right)+ \\
2^{n} \frac{B r}{B i}\left(\frac{3 n+1}{n}\right)^{n}
\end{gathered}
$$

The dimensionless average temperature $\theta_{a v}\left(x^{+}\right)$is calculated as:

$$
\begin{aligned}
& \theta_{a v}\left(x^{+}\right)= \int_{0}^{1} \theta\left(r^{+}, x^{+}\right) u^{+}\left(r^{+}\right) r^{+} d r^{+} \\
& \int_{0}^{1} u^{+}\left(r^{+}\right) r^{+} d r^{+} \\
&= \frac{2(3 n+1)}{(n+1)} \int_{0}^{1} \sum_{i=1}^{N} \beta_{i} M\left(a, b, \sigma_{i} r^{+2}\right) \times \\
& \exp \left(-\sigma_{i} \frac{r^{+2}}{2}\right) \cdot \exp \left(-\lambda_{i} x^{+}\right) u^{+}\left(r^{+}\right) r^{+} d r^{+}+ \\
& 2^{n} B r\left(\frac{n}{3 n+1}\right)^{1-n}\left[\frac{4 n^{2}+5 n+1}{2(n+1)(5 n+1)}\right]+2^{n} \frac{B r}{B i}\left(\frac{3 n+1}{n}\right)^{n}
\end{aligned}
$$

The local Nusselt number can be calculated from its definition by: 


$$
\begin{aligned}
N u & =2 \frac{k_{n f}}{k_{b f}} \frac{\left.\frac{\partial \theta}{\partial r^{+}}\right|_{r^{+}=1}}{\theta_{w}-\theta_{a v}} \\
& =2 \frac{k_{n f}}{k_{b f}} \frac{\sigma_{i \cdot}\left[\frac{2 a}{b} M\left(a+1, b+1, \sigma_{i}\right)-M\left(a, b, \sigma_{i}\right)\right] \exp \left(-\frac{1}{2} \sigma_{i}\right) \cdot \exp \left(-\lambda_{i} x^{+}\right)}{\theta_{w}-\theta_{a v}} \\
& -2 \frac{k_{n f}}{k_{b f}} \frac{2^{n-1} B r\left(\frac{3 n+1}{n}\right)^{n-1}}{\theta_{w}-\theta_{a v}}
\end{aligned}
$$

Using the initial few terms $(N=10) ; \beta_{i}, \sigma_{i}$ and $\lambda_{i}$ are determined and tabulated for three values of Biot number, $B i=2,20$ and 200 (see Tables 1, 2 and 3). Then, it is possible to directly calculate the temperatures and Nusselt number.

Table 1. Roots of transcendental Eq. (14) $\sigma_{i .}$, eigenvalues $\lambda_{i}$ and elements $\beta_{i}$, for $\boldsymbol{B} \boldsymbol{i}=\mathbf{2}$

\begin{tabular}{cccc}
\hline \multicolumn{3}{c}{$B i=2$} \\
\hline$i$ & $\sigma_{\text {. }}$ & $\lambda_{i}$ & $\beta_{i}$ \\
\hline $\mathbf{1}$ & 1.64125 & 3.119833 & 1.201287 \\
$\mathbf{2}$ & 5.478309 & 22.70115 & -0.2929295 \\
$\mathbf{3}$ & 9.435964 & 76.53324 & 0.1466777 \\
$\mathbf{4}$ & 13.41524 & 152.5915 & $-9.3045861 \mathrm{E}-02$ \\
$\mathbf{5}$ & 17.40259 & 415.2598 & $6.6288665 \mathrm{E}-02$ \\
$\mathbf{6}$ & 21.39392 & 3933.820 & $-5.0595514 \mathrm{E}-02$ \\
$\mathbf{7}$ & 25.38755 & 157098.3 & $4.0419348 \mathrm{E}-02$ \\
$\mathbf{8}$ & 29.38263 & 157098.3 & $-3.3354796 \mathrm{E}-02$ \\
$\mathbf{9}$ & 33.37869 & $1.0060228 \mathrm{E}+08$ & $2.8199114 \mathrm{E}-02$ \\
$\mathbf{1 0}$ & 37.37546 & $6.9776988 \mathrm{E}+09$ & $-2.4294296 \mathrm{E}-02$ \\
\hline
\end{tabular}

Table 2. Roots of transcendental Eq. (14) $\sigma_{i}$, eigenvalues $\lambda_{i}$ and elements $\beta_{i}$, for $\mathbf{B} \boldsymbol{i}=\mathbf{2 0}$

\begin{tabular}{cccc}
\hline \multicolumn{3}{c}{$B i=20$} \\
\hline$i$ & $\sigma_{\text {. }}$ & $\lambda_{i}$ & $\beta_{i}$ \\
\hline $\mathbf{1}$ & 2.516666 & 24.26892 & 1.436791 \\
$\mathbf{2}$ & 6.364460 & 86.76949 & -0.7173886 \\
$\mathbf{3}$ & 10.27052 & 86.76949 & 0.4871294 \\
$\mathbf{4}$ & 14.20003 & 155.4756 & -0.3694929 \\
$\mathbf{5}$ & 18.14348 & 843.8500 & 0.2971093 \\
$\mathbf{6}$ & 22.09643 & 87250.81 & -0.2478110 \\
$\mathbf{7}$ & 26.05635 & 87250.81 & 0.2119939 \\
$\mathbf{8}$ & 30.02160 & 5940566 & -0.1847850 \\
$\mathbf{9}$ & 33.99105 & $1.1995293 \mathrm{E}+09$ & 0.1634044 \\
$\mathbf{1 0}$ & 37.96392 & $3.8409449 \mathrm{E}+10$ & -0.1461825 \\
\hline
\end{tabular}

\subsection{Physical properties of the non Newtonian nanofluid}

This study adopts a single-phase approach supposing that the nanoparticles are uniformly dispersed into the base fluid. According to the mixing theory, the effective physical properties of nanofluids can be calculated using some formulations of density, nanofluid viscosity and specific heat capacity $[21,23]$ :

$$
\begin{gathered}
\rho_{n f}=(1-\varphi) \rho_{f}+\varphi \rho_{p} \\
\left(\rho c_{p}\right)_{n f}=(1-\varphi)\left(\rho c_{p}\right)_{f}+\varphi\left(\rho c_{p}\right)_{p}
\end{gathered}
$$

where, $\varphi$ is the volume fraction of nanoparticles and $\left(\rho c_{p}\right)_{n f}$ is the specific heat capacity of nanofluid.
Table 3. Roots of transcendental Eq. (14) $\sigma_{i .}$, eigenvalues $\lambda_{i}$ and elements $\beta_{i}$, for $\mathbf{B} \boldsymbol{i}=\mathbf{2 0 0}$

\begin{tabular}{cccc}
\hline & \multicolumn{3}{c}{$B i=200$} \\
\hline$i$ & $\sigma_{i .}$ & $\lambda_{i}$ & $\beta_{i}$ \\
$\mathbf{1}$ & 2.684267 & 7.018836 & 1.472802 \\
$\mathbf{2}$ & 6.643208 & 43.18540 & -0.7978859 \\
$\mathbf{3}$ & 10.62493 & 114.7704 & 0.5790848 \\
$\mathbf{4}$ & 14.61161 & 220.6732 & -0.4654360 \\
$\mathbf{5}$ & 18.60044 & 306.2844 & 0.3941488 \\
$\mathbf{6}$ & 22.59053 & 8565.360 & -0.3445711 \\
$\mathbf{7}$ & 26.58146 & 438140.6 & 0.3077402 \\
$\mathbf{8}$ & 30.57303 & 7893790 & -0.2791214 \\
$\mathbf{9}$ & 34.56509 & $7.8881824 \mathrm{E}+08$ & 0.2561094 \\
$\mathbf{1 0}$ & 38.55756 & $2.9010784 \mathrm{E}+10$ & -0.2371551 \\
\hline
\end{tabular}

\section{RESULTS AND DISCUSSIONS}

\subsection{Validation of the analytical solution}

In order to validate the developed analytical solution, the obtained results are first compared against those stated by Shah and London [25] for Newtonian fluid without effect of viscous dissipation $(B r=0)$ and $\varphi=0$. The comparison of the local Nusselt number from the canal inlet to the fully developed region is given in Table 4. As can be seen, the obtained results agree well with the above-mentioned reference. Moreover, according to the condition at the duct wall $\left(\frac{\partial \theta\left(x^{+}, r^{+}\right)}{\partial r^{+}}+\frac{1}{2} B i \theta=0\right)$, when Biot number is equal to zero, the particular insulated wall is obtained, and the wall subjected to constant temperature is achieved if Biot number tends to infinity. Consequently, as indicated in Table 1, the asymptotic Nusselt number approaches the known value corresponding to the constant heat flux $\left(\mathrm{Nu}_{\infty}=4.36\right)$ for low values of Biot number. However, for higher values of Biot number the Nusselt infinite value tends to the value related to a constant wall temperature $\left(\mathrm{Nu}_{\infty}=3.66\right)$.

Table 5 shows the similarity between the results obtained for the Nusselt infinite values $\left(\mathrm{Nu}_{\infty}\right)$ for non-Newtonian fluids taking into consideration the viscous dissipation effect and those obtained through the Barletta [26] correlation Eq. (48). The comparison reveals a specific agreement between the current process and the Eq. (48), reaffirming the robustness of the current theoritical solution. Other works treated this case $(\mathrm{Br} \neq 0)$, such as [27-30] for circular ducts, and in rectangular or parallel plates subjected to constant temperature at the wall $[31,32]$. The rheological behavior of nanofluids and heat transfer have been investigated [33, 34].

$$
N u_{\infty}=\frac{2(3 n+1)(5 n+1)}{n(4 n+1)}
$$

\subsection{The nanofluid $\left(\mathrm{TiO}_{2} / \mathrm{CMC} 0.5 \%\right.$ wt)}

The base fluid used in this work is a CMC solution of 0.5 $\mathrm{wt} \%$ in water. The analytical solution for $\mathrm{TiO}_{2}$ concentrations ranges from 0.5 to $4 \%$. The mixture $\mathrm{TiO}_{2} / \mathrm{CMC}$ employed behaves as non-Newtonian fluid (pseudo-plastic).

In our calculations, the parameters of the power-law model $(\mathrm{m}, \mathrm{n})$ obtained experimentally by Hojjat et al. [16, 17] for $\mathrm{TiO}_{2} / \mathrm{CMC}$-water, and the effective thermal conductivity at various concentrations $\varphi$, was used (see Table 6 and 7). 
Table 4. Local Nusselt number values at different axial points for Newtonian fluid $(\mathrm{Br}=0$ and $\varphi=0)$

\begin{tabular}{ccccccc}
\hline \multirow{2}{*}{$x^{+}$} & \multicolumn{2}{c}{$B i=2$} & \multicolumn{2}{c}{$B i=20$} & \multicolumn{2}{c}{$B i=200$} \\
\cline { 2 - 6 } & Present study & Shah et al. [25] & Present study & Shah et al. [25] & Present study & Shah et al. [25] \\
\hline $\mathbf{0 . 0 0 0 1}$ & 24.228 & 26.684 & 20.655 & 25.176 & 17.4952 & 22.936 \\
$\mathbf{0 . 0 0 0 2}$ & 14.073 & 21.190 & 11.320 & 19.884 & 11.237 & 18.001 \\
$\mathbf{0 . 0 0 0 5}$ & 12.694 & 15.556 & 10.444 & 14.350 & 10.590 & 13.084 \\
$\mathbf{0 . 0 0 1}$ & 11.397 & 12.297 & 9.484 & 11.227 & 10.120 & 10.300 \\
$\mathbf{0 . 0 0 2}$ & 9.820 & 9.745 & 8.354 & 8.804 & 7.901 & 8.145 \\
$\mathbf{0 . 0 0 5}$ & 7.708 & 7.249 & 6.532 & 6.471 & 6.058 & 6.061 \\
$\mathbf{0 . 0 1}$ & 6.342 & 5.899 & 5.106 & 5.236 & 4.958 & 4.954 \\
$\mathbf{0 . 0 2}$ & 5.311 & 4.945 & 4.120 & 4.388 & 4.210 & 4.196 \\
$\mathbf{0 . 0 5}$ & 4.432 & 4.263 & 3.771 & 3.838 & 3.728 & 3.722 \\
$\mathbf{0 . 1}$ & 4.166 & 4.133 & 3.762 & 3.763 & 3.670 & 3.666 \\
$\mathbf{0 . 2}$ & 4.125 & 4.124 & 3.762 & 3.763 & 3.668 & 3.666 \\
$\mathbf{0 . 5}$ & 4.125 & -- & 3.762 & -- & 3.668 & - \\
$\mathbf{1}$ & 4.125 & -- & 3.762 & -- & 3.668 & -- \\
\hline
\end{tabular}

Table 5. Fully developed Nusselt number for various fluids and Biot number $(B r \neq 0$ and $\varphi=0)$

\begin{tabular}{ccccccc}
\hline & \multicolumn{2}{c}{$B i=10^{6}$} & \multicolumn{2}{c}{$B i=100$} & \multicolumn{2}{c}{$B i=5$} \\
\hline $\boldsymbol{n}$ & Present study & Barletta et al. [26] & Present study & Barletta et al. [26] & Present study & Barletta et al. [26] \\
\hline $\mathbf{3}$ & 8.205 & 8.205 & 8.205 & 8.205 & 8.205 & 8.205 \\
$\mathbf{2}$ & 8.556 & 8.555 & 8.556 & 8.555 & 8.556 & 8.555 \\
$\mathbf{1 . 5}$ & 8.905 & 8.904 & 8.905 & 8.904 & 8.905 & 8.904 \\
$\mathbf{1}$ & 9.59 & 9.600 & 9.59 & 9.600 & 9.59 & 9.600 \\
$\mathbf{0 . 5}$ & 11.62 & 11.66 & 11.62 & 11.66 & 11.62 & 11.66 \\
$\mathbf{1 / 3}$ & 13.64 & 13.71 & 13.64 & 13.714 & 13.64 & 13.71 \\
\hline
\end{tabular}

Table 6. Power-law parameters and thermal conductivity of the worked nanofluid $[16,17]$

\begin{tabular}{cccc}
\hline$\varphi(\%)$ & $m$ & $n$ & $k_{n f}$ \\
\hline 0 & 0.145 & 0.542 & 0.600 \\
1 & 0.190 & 0.526 & 0.603 \\
3 & 0.240 & 0.502 & 0.641 \\
4 & 0.365 & 0.485 & 0.740 \\
\hline
\end{tabular}

Table 7. Fluid and nanoparticles thermophysical properties [35]

\begin{tabular}{cccc}
\hline Propriétés & $\boldsymbol{\rho}\left(\boldsymbol{k g} / \boldsymbol{m}^{\mathbf{3}}\right)$ & $\boldsymbol{C p}(\mathbf{j} / \boldsymbol{k g} \boldsymbol{K})$ & $\boldsymbol{k}(\boldsymbol{W} / \boldsymbol{m} . \boldsymbol{K})$ \\
\hline water & 998.2 & 4185 & 0.602 \\
CMC & 1002 & 4500 & 0.6 \\
Titanium oxide $\left(\mathrm{TiO}_{2}\right)$ & 4250 & 686.2 & 8.9538 \\
\hline
\end{tabular}

\subsection{Effect of Biot number on distribution of bulk and wall temperatures}

Figure 2 depicts the distributions of temperatures for three values of Biot number $(\mathrm{Bi}=0.5,5$ and 50$)$, and different Brinkman numbers at $\varphi=0 \%(\mathrm{n}=0.542)$. It can be observed that the difference between the wall and the bulk temperature is very small everywhere along the duct for a low Biot number $(B i=0.5)$, especially at the entrance region for all Brinkman number values (see Figure 2 (a)). In fact, when Bi tends to zero, the duct wall is nearly insulated and there is negligible thermal energy transferred to the ambient medium. For higher Biot number values $(B i=50)$, a noticeable difference between temperatures is underlined at the entrance region where the effects of $\mathrm{Br}$ number are negligible (Figure 2(c)). However, the dimensionless bulk and wall temperature in the thermally developed region tend to have an asymptotic value depending on the cooling or heating condition. The results reported display an obvious dependency of the asymptotic wall temperature on $\mathrm{Br}$ number. In addition, the wall and bulk temperature can be observed to decrease speedily along the duct as Biot number increases due to the growth in the external heat transfer. For other values of volume concentration $\varphi$, the same behavior of the wall and bulk temperature distribution is observed. At a same value of Brinkman number, the asymptotic values of wall and bulk temperature increase when Biot number decreases, which can be explained by the low thermal energy transferred to external medium.

\subsection{Brinkman number effect on temperature distributions}

Figure 2(a) shows the effects of viscous dissipation. In the cooling case $(B r>0)$ and for a low value of Biot number $(B i=0.5)$, it is observed that the temperature of fluid becomes greater than the temperature in the inlet duct $T_{e}$, which can be explained firstly by the heat produced by the viscous dissipation effect stored in fluid, and secondly, by the weak heat exchange with the external environment. In addition, the asymptotic values of bulk and wall temperature decrease when $\mathrm{Br}$ decreases. In the heating case $(B r<0)$, knowing that the fluid temperature at the duct inlet is lower than the external temperature $T_{e}<T_{\infty}$, and due to the energy produced by viscous dissipation, and the thermal exchange with the external medium is weak, the mean and wall temperatures become higher than the temperature $T_{\infty}$ and take negative values. Also, the asymptotic values decrease as Brinkman number decreases. Figures 2(b) and 2(c) show the cases of $B i=5$ and 50, where the heat transferred with the external medium is improved. The nanofluid temperature exceeds the external temperature in the heating case $(\mathrm{Br}<0)$ and the opposite appears in the cooling case $(B r>0)$.

\subsection{Effect of volume concentration on bulk temperature distributions}

Figure 3 shows the progression of the bulk temperature $\left(\theta_{a v}\right)$ along the duct at different nanoparticles concentrations with Biot number equal to 0.5 . Two cases are considered, the 
cooling case corresponding to $B r=1$ (Figure 3(a)) and the heating case related to $B r=-1$ (Figure 3(b)). In the first case, due to the heat accumulated in fluid and low heat exchanged with the ambient, the average temperature is greater than the external temperature. As seen, when the volume concentration increases, the asymptotic value of the average temperature decreases, which can be explained by the increase of heat transfer due to the addition of nanoparticles. In the heating case, the bulk temperature is less than the external temperature. The asymptotic value increases when the volume concentration increases (Figure 3(b)). Remember that the mixture $\mathrm{TiO}_{2}-\mathrm{CMC}$ behaves as a shear thinning nonNewtonian nanofluid $(n<1)$ and the consistency factor, $m$ (equivalent to a viscosity for a Newtonian fluid) increases with nanoparticles loading (see Table 6). Moreover, the velocity field is influenced by the behaviour index variation and has a significant gradient in the proximity of the wall, thus enhancing heat transfer rate between the wall and the fluid. In Figure 4, the bulk temperature, $\theta_{a v}$ for $B i=5$ is lower than the temperature of external medium related to the enhancement of heat transfer from the wall. Furthermore, the asymptotic value decreases when the concentration increases in the cooling case (Figure 4(a), $B r=1$ ), and the opposite occurs in the heating case (Figure 4(b), $B r=-1$ ). The same behavior is noted for $B i$ $=50$ and 500 (see Figures 5 and 6 ). To illustrate the influence of Biot number at the fixed concentration, Figure 7 demonstrates the variation of the average temperature for $\varphi=1 \%, B r=1$ and -1 . As it can be seen, when $B i$ increases the asymptotic value decreases in the cooling case $(B r>0)$ and increases in the heating case $(B r<0)$. This can be explained by the growing heat transfer from the wall when Biot increases.
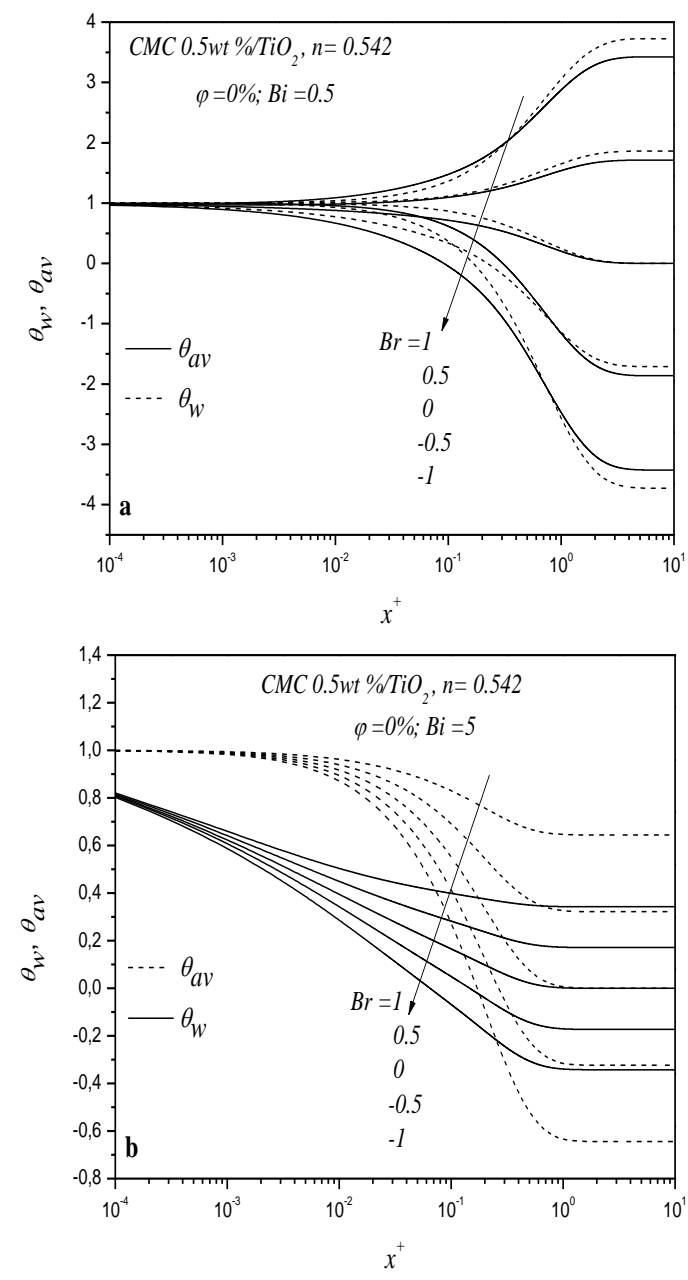

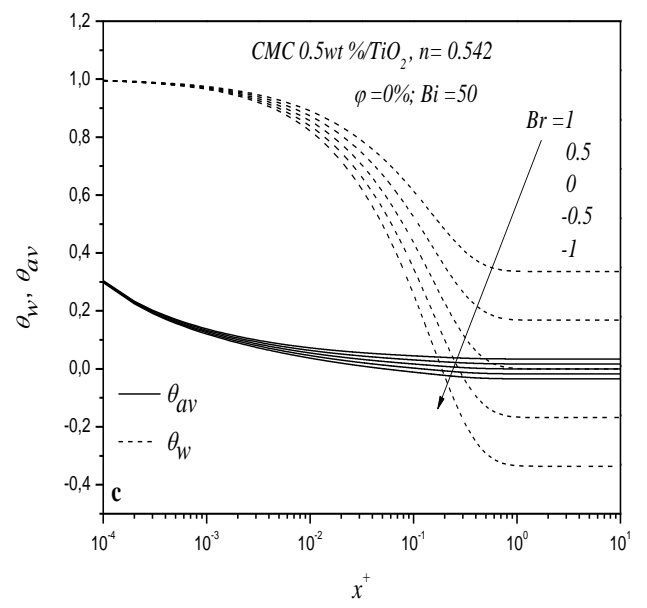

Figure 2. Effect of Biot and Brinkman numbers on distribution of $\theta_{w}, \theta_{a v},(\varphi=0 \%)$
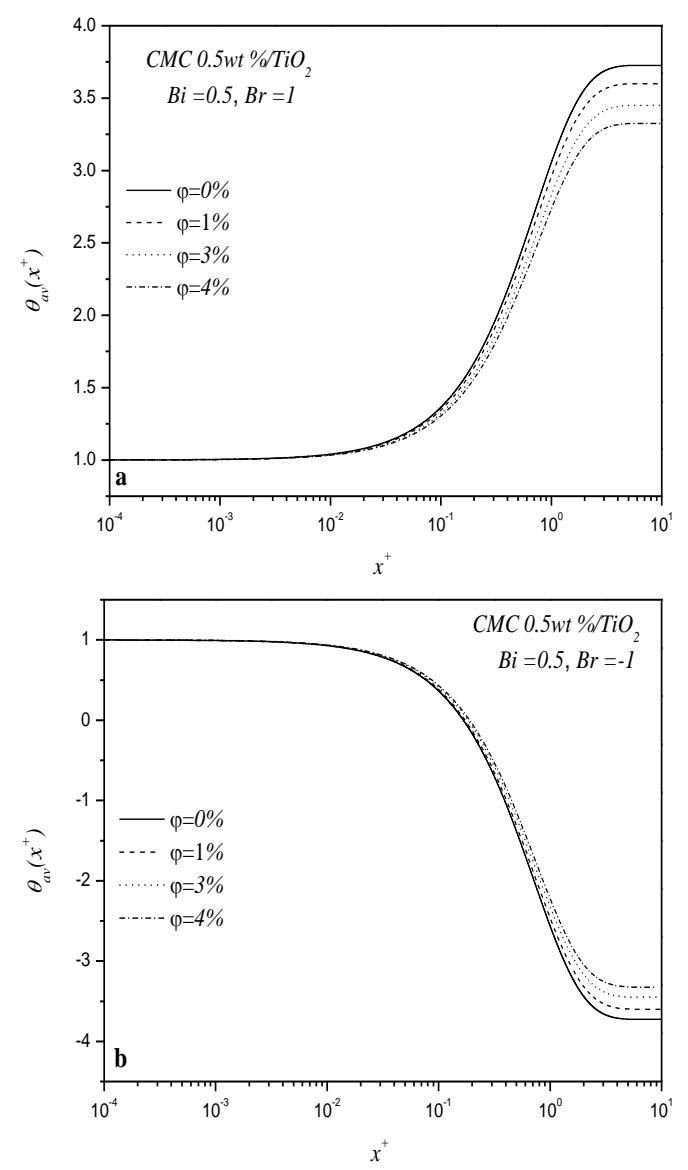

Figure 3. Effect of concentration $\varphi$ and $B r$ on distribution of $\theta_{a v}$. (a): $B r=1$; (b): $B r=-1$

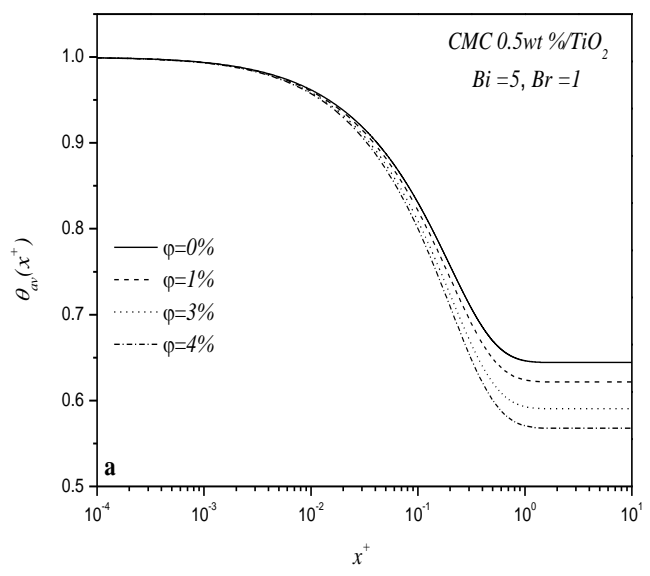




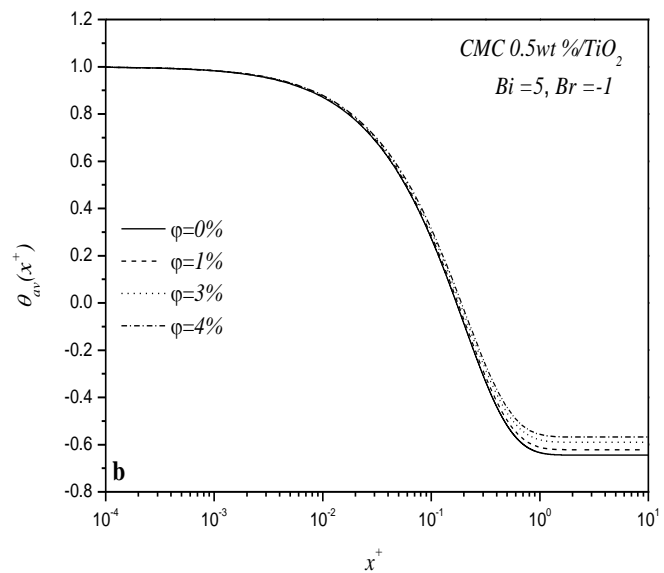

Figure 4. Effect of $\varphi$ and $B r$ on distribution of $\theta_{a v}, B i=5$
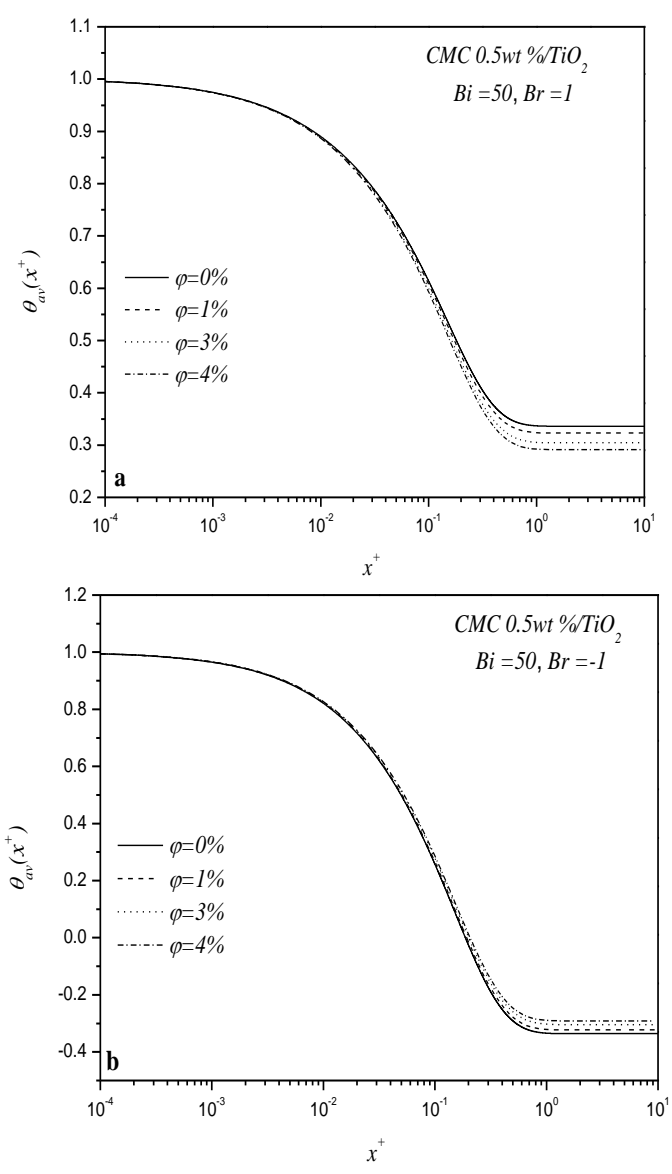

Figure 5. Effect of $\varphi$ and $B r$ on distribution of $\theta_{a v}, B i=50$

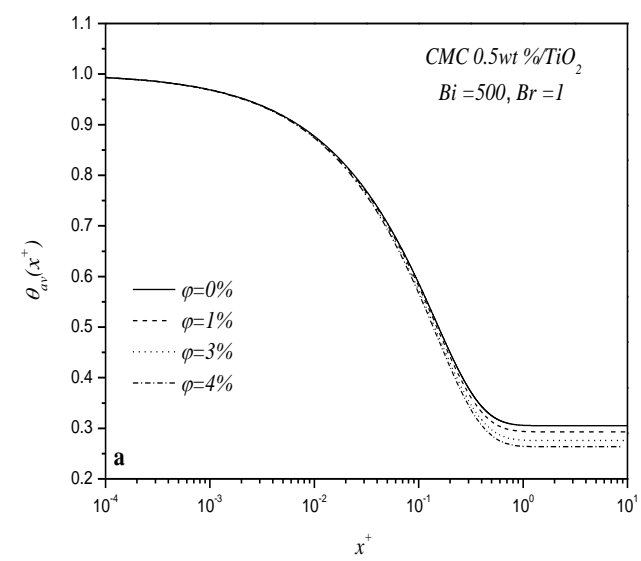

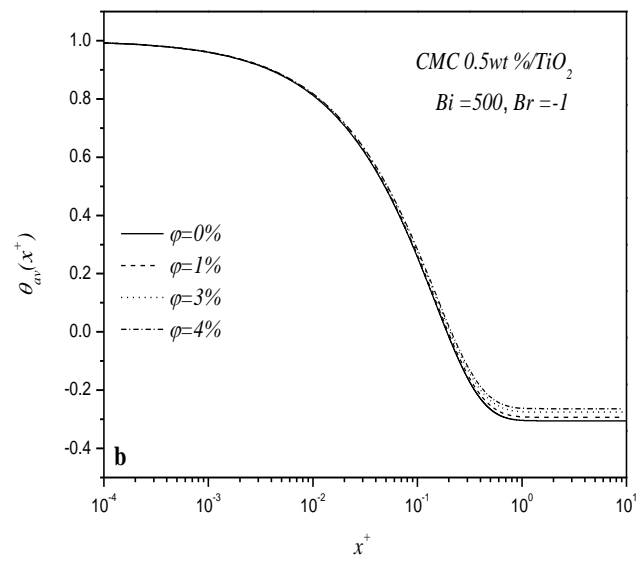

Figure 6. Effect of $\varphi$ and $B r$ on distribution of $\theta_{a v}, B i=500$
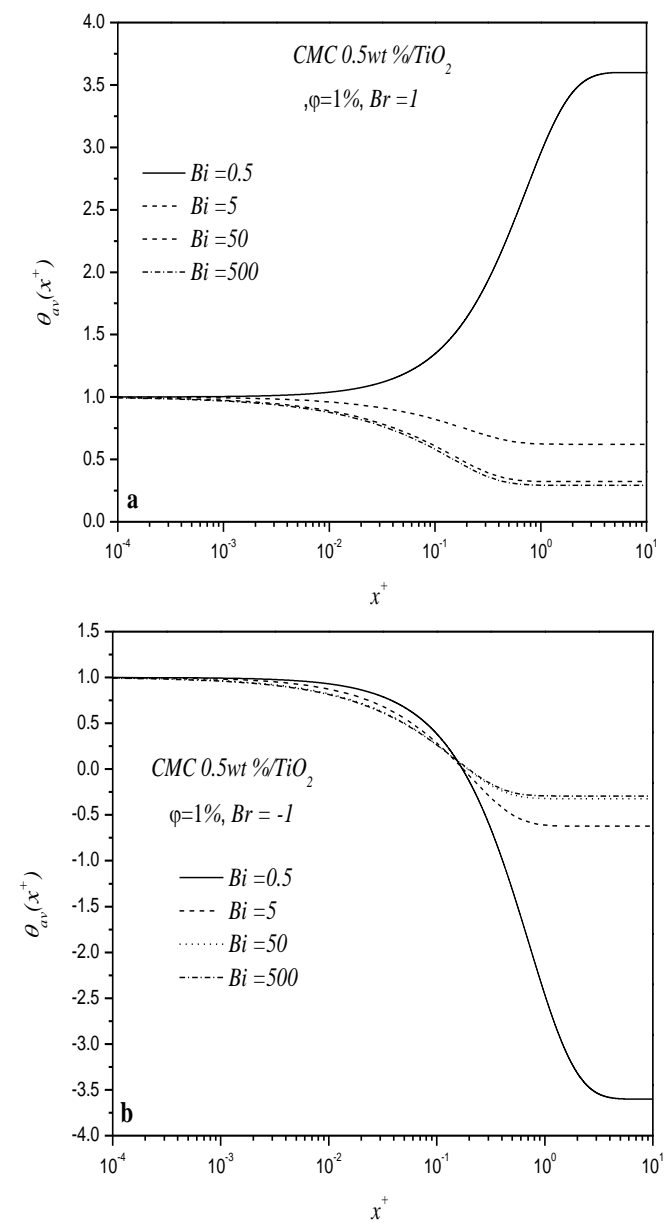

Figure 7. Effect of Biot number on distribution of $\theta_{a v}$, (a): $B r=1$; (b): $B r=-1$

\subsection{Effect of Biot, Brinkman numbers on the evolution of local $\mathrm{Nu}(\mathrm{x})$}

Figure 8 illustrates the evolution of Nusselt number along the duct for different Brinkman and Biot numbers. As it can be seen, a singular point in heating cases $(B r<0)$ is observed in the developing zone, where Nusselt local changes from $-\infty$ to $+\infty$. This singular point corresponds exactly to the position where the bulk and wall temperature intersected (see Figures 2). At a fixed Biot number, this point position shifts closer to $x^{+}=0$ when Brinkman number decreases. For a fixed Brinkman number $B r=-1$, the position of a single point is shifting closer to the entrance region when Biot number decreases due to the 
low heat transfer across the duct wall. For low value of Biot number $(B i=0.5)$, the axial evolution of Nusselt number shows a peak for $B r=0.5$ and $B r=1$. This highest point is developed to create a single point where Nusselt number tends to infinity because the heat generated by viscose dissipation is more significant. $N u$ increases when the volume concentration increases (see Figure 9). In fact, asymptotic value of Nusselt number increases from 11.34 to 14.54 when $\varphi$ increases from 0 to $4 \%$ (see Figure 10). Utilizing non-linear regression technique, an approximated formula for the asymptotic Nusselt number with respect to the concentration of $\mathrm{TiO}_{2}$ in CMC $0.5 \%$ wt non-Newtonian base fluid is obtained (Rsquared value 0.91 ) as:

$$
N u_{\infty}=11.34+42.66 \varphi-3.6 \times 10^{3} \varphi^{2}+11.33 \times 10^{4} \varphi^{3}
$$

The proposed correlation is valid for $0 \leq \varphi \leq 4 \%$ and the committed deviation is lower than $1 \%$ in most cases.
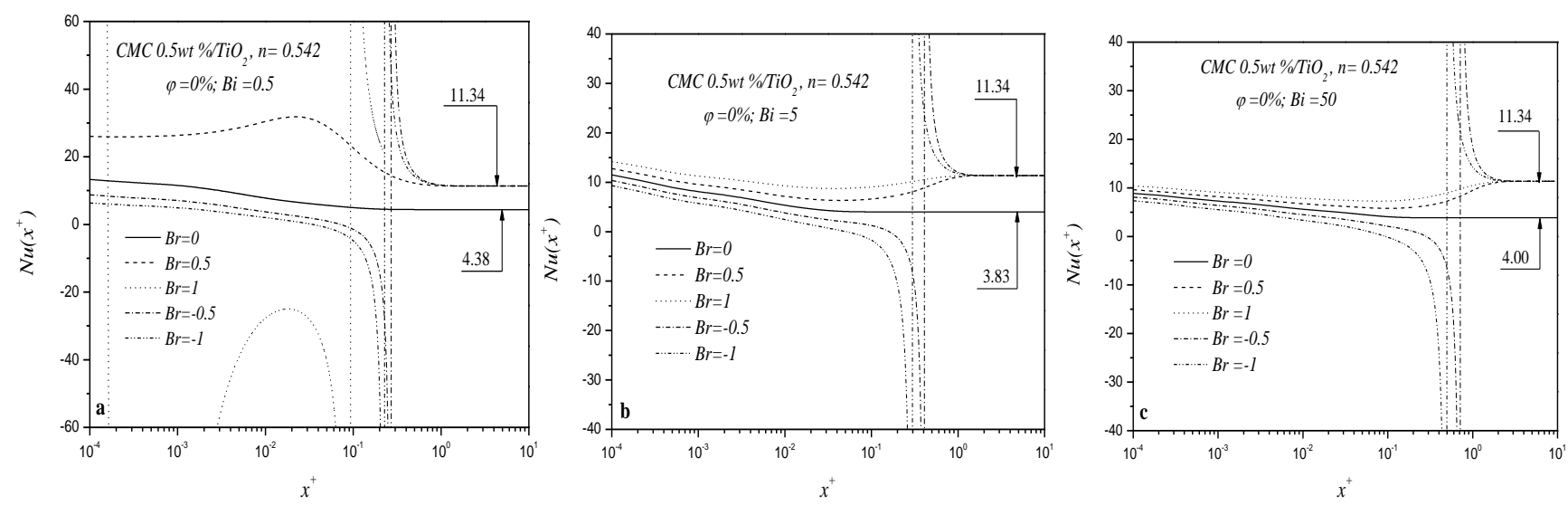

Figure 8. Effect of $B i$ and $B r$ numbers on the local Nusselt number $(\varphi=0 \%)$
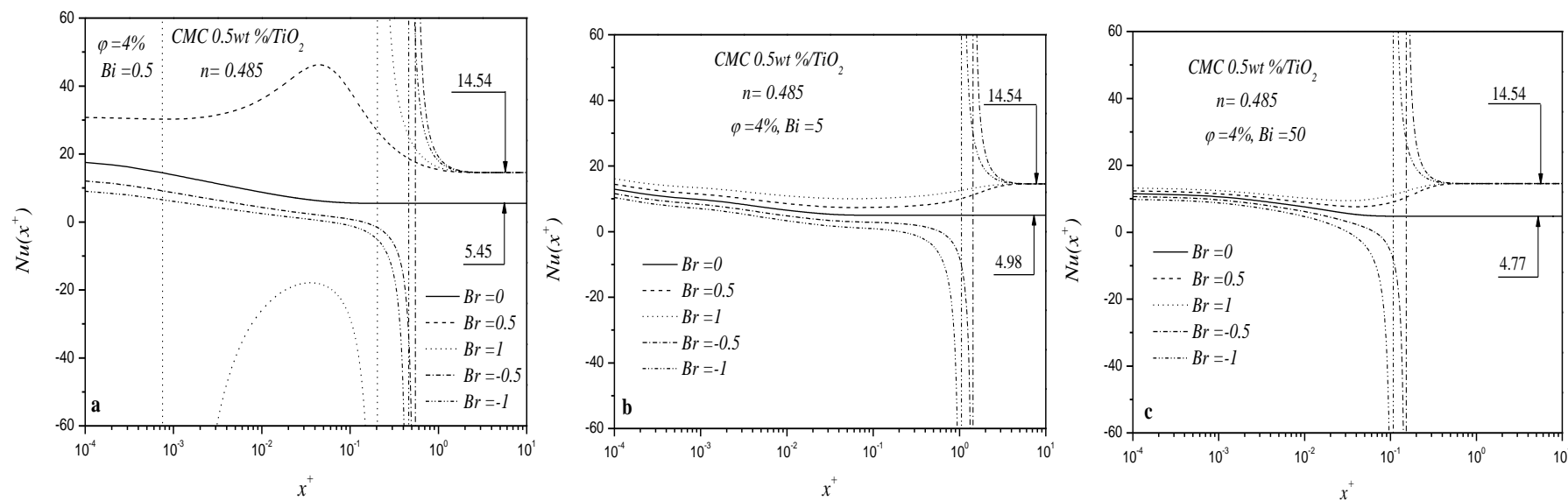

Figure 9. Effect of $B i$ and $B r$ numbers on the local Nusselt number $(\varphi=4 \%)$

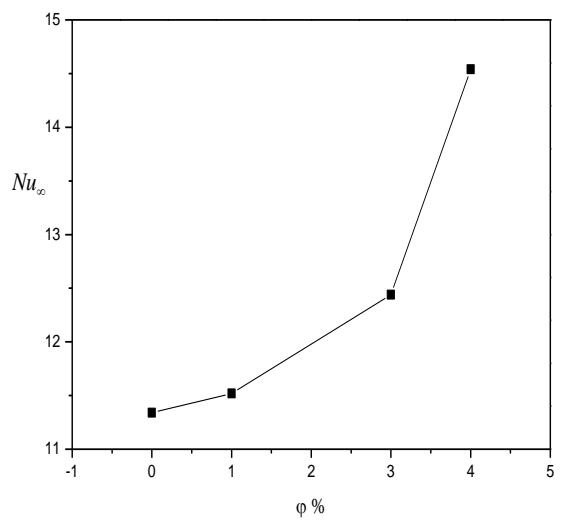

Figure 10. Asymptotic Nusselt number at various concentrations

\subsection{Influence of the Peclet number on $\mathrm{Nu}\left(\mathrm{x}^{*}\right)$ evolution}

Figures 11 to 14 show the evolution of local Nusselt number
$N u\left(x^{*}\right)$ under the effects of Peclet number at $B r=0.5$ and -0.5 , with Biot number taken equal to $B i=0.5$ and 50 . Therefore, the dimensionless axial coordinate is given by: $x^{*}=x / R$. The Peclet number covering the range [300-1000] for $\varphi=0$ and $4 \%$ at $B r=0.5$ and -0.5 . As can be observed in the cooling case $(B r=0.5)$ and in the case of $B i=0.5$, there was a peak in the thermally developing zone, and Nusselt number decreases continuously along the duct to an asymptotic value that is independent of $P e_{n f}$ (see Figures 11(a) and 13(a)). This peak is due to the very small difference between the wall and average temperatures caused by the heat generated by viscous dissipation. When the $P e_{n f}$ number reduces, the point position shifts are near to $x^{*}=0$. The amount of this peak is high as the volume concentration increases. For $B i=50$, Nusselt number has a minimum value in the thermally developing region. This minimum value is caused by the important difference between the $\theta_{a v}$ and $\theta_{w}$ temperatures due to the improvement in heat transfer with the external medium. In the heating case $(B r=-$ $0.5)$, Nusselt number tends to $\pm \infty$ where the bulk temperature of nanofluid becomes equal to the temperature at the wall as 
seen in Figures 12(b) and 14(b). Another suitable characteristic of these figures is that with increasing nanoparticles concentration $\varphi$, the asymptotic Nusselt number increases. The peak improvement is obtained at the high concentration value of $4 \%$ where, $N u_{\infty}$ increases from 11.34 to 14.54 .
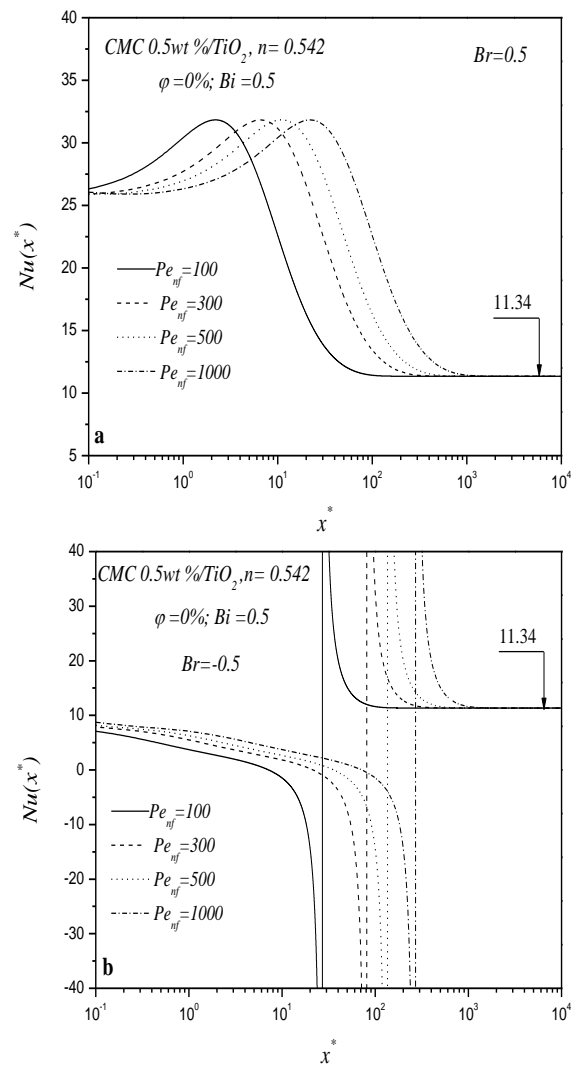

Figure 11. Axial evolution of Nusselt number at various $P e_{n f}$ number ( $\varphi=0 \%, B i=0.5$ ), (a) $B r=0.5$, (b) $B r=-0.5$
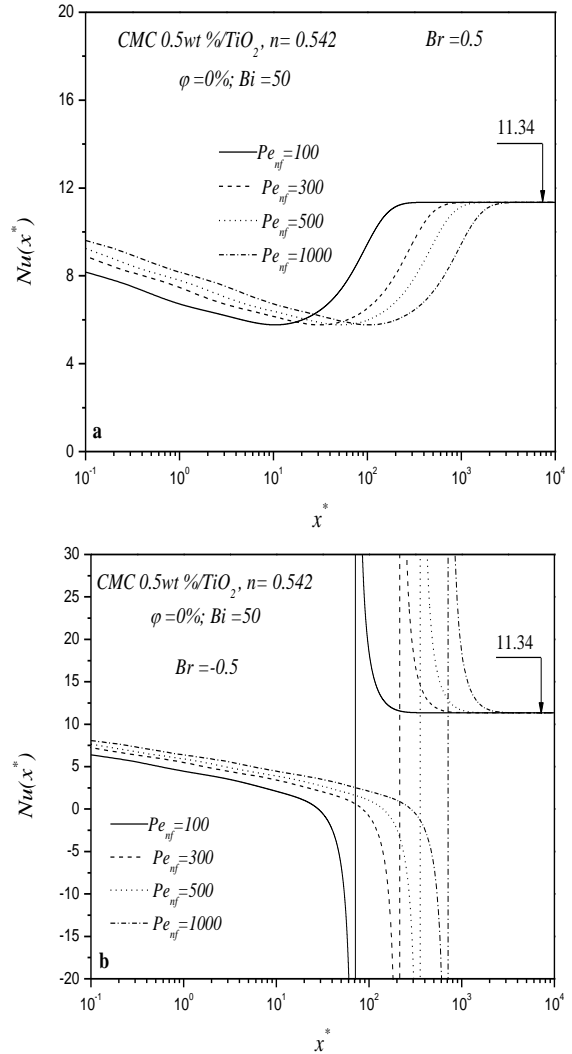

Figure 12. Axial evolution of Nusselt number at various $P e_{n f}$ number ( $\varphi=0 \%, B i=50)$, (a) $B r=0.5$, (b) $B r=-0$
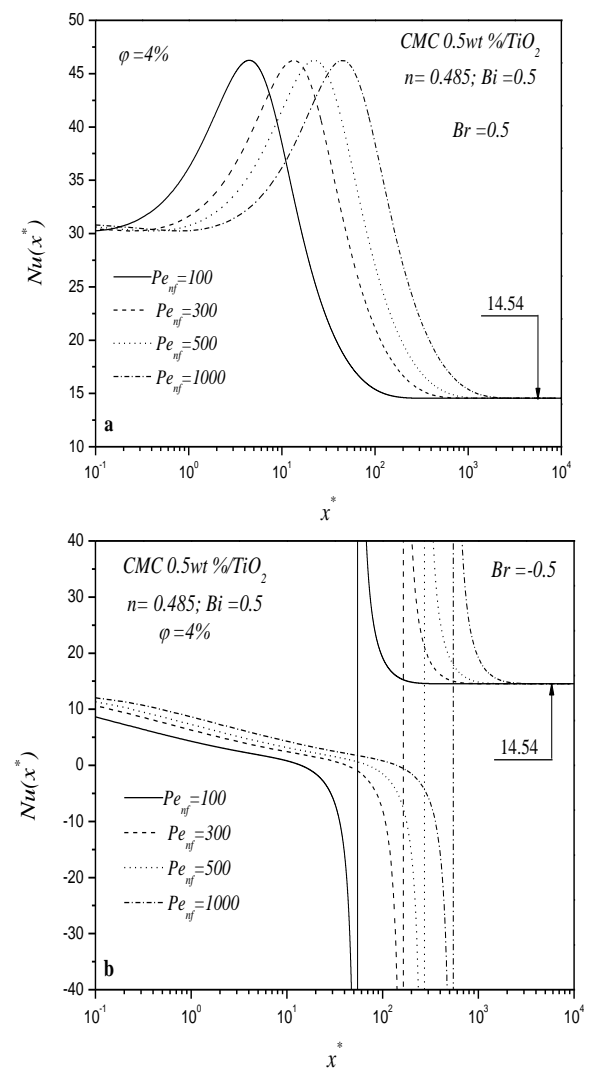

Figure 13. Axial evolution local Nusselt number for various $P e_{n f}$ number $(\varphi=4 \%, B i=0.5)$, (a) $B r=0.5$, (b) $B r=-0.5$
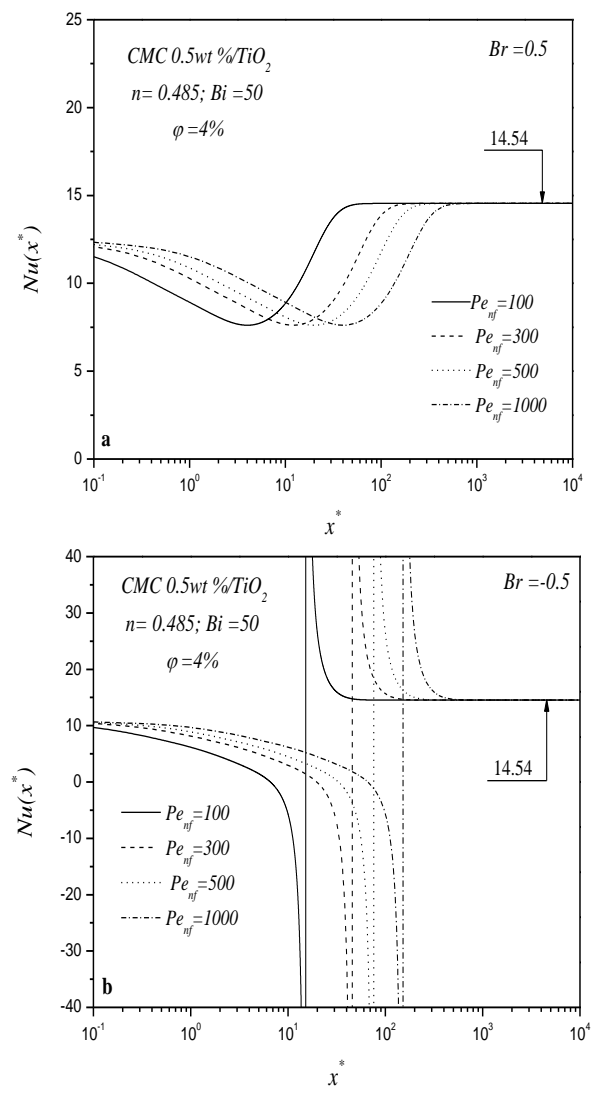

Figure 14. Axial evolution of Nusselt number for various $P e_{n f}$ number $(\varphi=4 \%, B i=50$ ), (a) $B r=0.5$, (b) $B r=-0.5$ 


\section{CONCLUSION}

Analytical solution for laminar convective heat transfer of non-Newtonian nanofluid in circular duct under convective boundary condition (third kind) is performed using the variational method of Ritz-Laplace. This solution is validated in literature on the specific cases available. The effect of Biot, Brinkman, Peclet number and concentration $(\varphi)$ on the Nusselt number evolutions are given graphically. The bulk and wall temperatures show an asymptotic value depending on Brinkman, Biot numbers and volume concentration $\varphi$.

In the case of low value of the Biot number $(B i=0.5)$, and at a fixed value of Brinkman number, when the volume concentration $\varphi$ increases, the asymptotic value of the bulk temperature decreases in the cooling case $(B r>0)$ while it increases in the heating case $(B r<0)$. Furthermore, the obtained results show clearly that the addition of $\mathrm{TiO}_{2}$ nanoparticles produce an important improvement in heat transfer compared to base fluid ( $0.5 \mathrm{wt} \%$ of CMC in water). Besides, it's found that when $\varphi$ rises from 0 to $4 \%$, the asymptotic value of Nusselt number increases from 11.34 to 14.54 , independently of Biot, Brinkman and Peclet numbers.

\section{REFERENCES}

[1] Fabbri, G. (1999). Optimum profiles for asymmetrical longitudinal fins in cylindrical ducts. International Journal of Heat and Mass Transfer, 42(3): 511-523. https://doi.org/10.1016/S0017-9310(98)00179-3

[2] Ouzzane, M., Galanis, N. (2001). Numerical analysis of mixed convection in inclined tubes with external longitudinal fins. Solar Energy, 71(3): 199-211. https://doi.org/10.1016/S0038-092X(01)00030-5

[3] Barba, A., Rainieri, S., Spiga, M. (2002). Heat transfer enhancement in a corrugated tube. International Communications in Heat and Mass Transfer, 29(3): 313322. https://doi.org/10.1016/S0735-1933(02)00321-4

[4] Ragueb, H., Mansouri, K. (2018). An analytical study of the periodic laminar forced convection of nonNewtonian nanofluid flow inside an elliptical duct. International Journal of Heat and Mass Transfer, 127: 469-483.

https://doi.org/10.1016/j.ijheatmasstransfer.2018.07.051

[5] Choi, S.U., Eastman, J.A. (1995). Enhancing thermal conductivity of fluids with nanoparticles (No. ANL/MSD/CP-84938; CONF-951135-29). Argonne National Lab., IL (United States).

[6] Shahrul, I.M., Mahbubul, I.M., Khaleduzzaman, S.S., Saidur, R., Sabri, M.F.M. (2014). A comparative review on the specific heat of nanofluids for energy perspective. Renewable and Sustainable Energy Reviews, 38: 88-98. https://doi.org/10.1016/j.rser.2014.05.081

[7] Bahiraei, M., Hangi, M. (2015). Flow and heat transfer characteristics of magnetic nanofluids: A review. Journal of Magnetism and Magnetic Materials, 374: 125-138. https://doi.org/10.1016/j.jmmm.2014.08.004

[8] Ashrafmansouri, S.S., Esfahany, M.N. (2014). Mass transfer in nanofluids: A review. International Journal of Thermal Sciences, 82: 84-99. https://doi.org/10.1016/j.ijthermalsci.2014.03.017

[9] Sidik, N.A.C., Mohammed, H.A., Alawi, O.A., Samion, S. (2014). A review on preparation methods and challenges of nanofluids. International Communications in Heat and Mass Transfer, 54: 115-125. https://doi.org/10.1016/j.icheatmasstransfer.2014.03.00 2

[10] Celen, A., Çebi, A., Aktas, M., Mahian, O., Dalkilic, A.S., Wongwises, S. (2014). A review of nanorefrigerants: flow characteristics and applications. International Journal of Refrigeration, 44: 125-140. https://doi.org/10.1016/j.ijrefrig.2014.05.009

[11] Mahian, O., Kianifar, A., Kalogirou, S.A., Pop, I., Wongwises, S. (2013). A review of the applications of nanofluids in solar energy. International Journal of Heat and Mass Transfer, 57(2): 582-594. https://doi.org/10.1016/j.ijheatmasstransfer.2012.10.037

[12] Lee, S., Choi, S.U.S., Li, S., Eastman, J.A. (1999). Measuring thermal conductivity of fluids containing oxide nanoparticles. Trans ASME J. Heat Transfer, 9: 121-280. https://doi.org/10.1115/1.2825978

[13] Heris, S.Z., Etemad, S.G., Esfahany, M.N. (2006). Experimental investigation of oxide nanofluids laminar flow convective heat transfer. International Communications in Heat and Mass Transfer, 33(4): 529535 . https://doi.org/10.1016/j.icheatmasstransfer.2006.01.00 5

[14] Heris, S.Z., Esfahany, M.N., Etemad, S.G. (2007). Experimental investigation of convective heat transfer of $\mathrm{Al}_{2} \mathrm{O}_{3}$ /water nanofluid in circular tube. International Journal of Heat and Fluid Flow, 28(2): 203-210. https://doi.org/10.1016/j.ijheatfluidflow.2006.05.001

[15] Santra, A.K., Sen, S., Chakraborty, N. (2009). Study of heat transfer due to laminar flow of copper-water nanofluid through two isothermally heated parallel plates. International Journal of Thermal Sciences, 48(2): 391400. https://doi.org/10.1016/j.ijthermalsci.2008.10.004

[16] Hojjat, M., Etemad, S.G., Bagheri, R., Thibault, J. (2011). Convective heat transfer of non-Newtonian nanofluids through a uniformly heated circular tube. International Journal of Thermal Sciences, 50(4): 525-531. https://doi.org/10.1016/j.ijthermalsci.2010.11.006

[17] Hojjat, M., Etemad, S.G., Bagheri, R., Thibault, J. (2011). Thermal conductivity of non-Newtonian nanofluids: experimental data and modeling using neural network. International Journal of Heat and Mass Transfer, 54(5-6): 1017-1023. https://doi:10.1016/j.ijheatmasstransfer.2010.11.039

[18] Hojjat, M., Etemad, S.G., Bagheri, R., Thibault, J. (2011). Rheological characteristics of non-Newtonian nanofluids: experimental investigation. International Communications in Heat and Mass Transfer, 38(2): 144148. https://doi.org/10.1016/j.icheatmasstransfer.2010.11.01 9

[19] Yang, Y., Zhang, Z.G., Grulke, E.A., Anderson, W.B., $\mathrm{Wu}, \mathrm{G}$. (2005). Heat transfer properties of nanoparticlein-fluid dispersions (nanofluids) in laminar flow. International Journal of Heat and Mass Transfer, 48(6): 1107-1116. https://doi.org/10.1016/j.ijheatmasstransfer.2004.09.038

[20] Bahiraei, M., Mazaheri, N., Alighardashi, M. (2017). Development of chaotic advection in laminar flow of a non-Newtonian nanofluid: a novel application for efficient use of energy. Applied Thermal Engineering, 124:

1213-1223. https://doi.org/10.1016/j.applthermaleng.2017.06.106 
[21] Tahiri, A., Mansouri, K. (2017). Theoretical investigation of laminar flow convective heat transfer in a circular duct for a non-Newtonian nanofluid. Applied Thermal Engineering, 112: 1027-1039. https://doi:10.1016/j.applthermaleng.2016.10.137

[22] Tahiri, A., Mansouri, K. (2018). Analytical solution by Laplace-ritz variational method for non-Newtonian nanofluid inside a circular tube. International Journal of Mechanical Sciences, 135: 596-608. https://doi.org/10.1016/j.ijmecsci.2017.12.006

[23] Ragueb, H., Mansouri, K. (2019). Numerical investigation of laminar forced convection for a nonNewtonian nanofluids flowing inside an elliptical duct under convective boundary condition. International Journal of Numerical Methods for Heat \& Fluid Flow, 29(1): 334-364. https://doi.org/10.1108/HFF-02-20180055

[24] Cotta, R.M., Özi, M.N. (1986). Laminar forced convection of power-law non-Newtonian fluids inside ducts. Wärme-und Stoffübertragung, 20(3): 211-218. https://doi.org/10.1007/BF01303453

[25] Shah, R.K., London, A.L. (2014). Laminar flow forced convection in ducts: A source book for compact heat exchanger analytical data. Academic Press.

[26] Barletta, A. (1997). Slug flow heat transfer in circular ducts with viscous dissipation and convective boundary conditions. International Journal of Heat and Mass Transfer, 40(17): 4219-4228. https://doi.org/10.1016/s0017-9310(97)00069-0

[27] Barletta, A., Magyari, E. (2007). Forced convection with viscous dissipation in the thermal entrance region of a circular duct with prescribed wall heat flux. International Journal of Heat and Mass Transfer, 50(1-2): 26-35. https://doi.org/10.1016/j.ijheatmasstransfer.2006.06.036

[28] Tso, C.P., Sheela-Francisca, J., Hung, Y.M. (2010). Viscous dissipation effects of power-law fluid flow within parallel plates with constant heat fluxes. Journal of Non-Newtonian Fluid Mechanics, 165(11-12): 625630. https://doi:10.1016/j.jnnfm.2010.02.023

[29] Aydin, O. (2005). Effects of viscous dissipation on the heat transfer in forced pipe flow. Part 1: Both hydrodynamically and thermally fully developed flow. Energy Conversion and Management, 46(5): 757-769. https://doi.org/10.1016/j.enconman.2004.05.004

[30] Hartnett, J.P., Kostic, M. (1989). Heat transfer to Newtonian and non-Newtonian fluids in rectangular ducts. In Advances in Heat Transfer, 19: 247-356.

[31] Barletta, A. (1996). Fully developed laminar forced convection in circular ducts for power-law fluids with viscous dissipation. International Journal of Heat and Mass $\quad$ Transfer, 40(1): 15-26. https://doi.org/10.1016/S0017-9310(96)00094-4

[32] Jambal, O., Shigechi, T., Davaa, G., Momoki, S. (2005). Effect of viscous dissipation and axial heat conduction on heat transfer for non-Newtonian fluids in ducts with uniform wall temperature, Part I. Parallel plates and circular ducts. Int. Commun. Heat Mass Transfer, 32: $1165-1173$.

https://doi.org/10.1016/j.icheatmasstransfer.2005.07.00 3

[33] Ding, Y., Alias, H., Wen, D., Williams, R.A. (2006). Heat transfer of aqueous suspensions of carbon nanotubes (CNT nanofluids). International Journal of
Heat and Mass Transfer, 49(1-2): 240-250. https://doi.org/10.1016/j.ijheatmasstransfer.2005.07.009

[34] Mahbubul, I.M., Khaleduzzaman, S.S., Saidur, R., Amalina, M.A. (2014). Rheological behavior of $\mathrm{Al}_{2} \mathrm{O}_{3} / \mathrm{R} 141 \mathrm{~b}$ nanorefrigerant. International Journal of Heat and Mass Transfer, 73: 118-123. https://doi.org/10.1016/j.ijheatmasstransfer.2014.01.073

[35] Islami, S.B., Dastvareh, B., Gharraei, R. (2014). An investigation on the hydrodynamic and heat transfer of nanofluid flow, with non-Newtonian base fluid, in micromixers. International Journal of Heat and Mass Transfer, 78: 917-929. https://doi.org/10.1016/j.ijheatmasstransfer.2014.07.022

\section{NOMENCLATURE}

[ $\left.A^{*}\right] \quad$ Modified matrix

$a_{i j}^{*} \quad$ Modified matrix elements

$b_{i} \quad$ Vector $[b]$ elements

$\mathrm{Br} \quad$ Brinkman number

$\mathrm{Bi} \quad$ Biot number

[C] Column vector of unknown values $C_{i}$

$C p \quad$ Specific heat capacity

$d_{i j} \quad$ Matrix $[D]$ elements

I Variational form

$k \quad$ Thermal conductivity

$m \quad$ Consistency coefficient

$n \quad$ Power-law index

$\mathrm{Nu} \quad$ Nusselt number

$\mathrm{Pe} \quad$ Peclet number

$r \quad$ Radial coordinate

$r^{+} \quad$ Dimensionless radial coordinate $[r / R]$

$R \quad$ Circular duct radius

$s \quad$ Transform variable of Laplace

$T \quad$ Dimensional fluid temperature

$T_{e} \quad$ Inlet temperature

$T_{\mathrm{w}} \quad$ Wall temperature

$u(r) \quad$ Velocity profile of fully developed flow

$u_{a v} \quad$ Average velocity of flow

$x \quad$ Axial coordinate

$x^{+} \quad$ Dimensionless axial coordinate

\section{Greek symbols}

$\alpha \quad$ Thermal diffusivity

$\beta_{l} \quad$ Vector $[b][D]^{-1}$ element

$\varphi \quad$ Concentration of nanoparticles

$\mu \quad$ Fluid dynamic viscosity

$\rho \quad$ Density

$\theta \quad$ Profile of dimensionless temperature $\theta=\left[\frac{T-T_{\infty}}{T_{e}-T_{\infty}}\right]$

$\psi_{i} \quad$ Hypergeometric function

\section{Subscriptsand superscripts}

$a v, e, \quad$ Average, entrance and wall

$w$

$f, n f, p$ Fluid, nanofluid and particle

+ , - Dimensionless parameters and Laplace transformed function 